\title{
Characterization of Brain Development in the Ferret via MRI
}

\author{
ALAN R. BARNETTE, JEFFERY J. NEIL, CHRISTOPHER D. KROENKE, JENNIFER L. GRIFFITH, ADRIAN A. EPSTEIN, \\ PHILIP V. BAYLY, ANDREW K. KNUTSEN, AND TERRIE E. INDER
}

\begin{abstract}
Department of Pediatrics [A.R.B., J.J.N., T.E.I.]; Mallinckrodt Institute of Radiology [J.J.N., J.L.G., A.A.E., P.V.B., A.K.K., T.E.I.]; and Department of Neurology [J.J.N., T.E.I.], Washington University, St. Louis, Missouri 63110; Department of Behavioral Neuroscience [C.D.K.], Oregon Health and Science University, Portland, Oregon 97239; Department of Mechanical Engineering [P.V.B., A.K.K.], Washington University, St. Louis, Missouri 63105
\end{abstract}

\begin{abstract}
Animal models with complex cortical development are useful for improving our understanding of the wide spectrum of neurodevelopmental challenges facing human preterm infants. MRI techniques can define both cerebral injury and alterations in cerebral development with translation between animal models and the human infant. We hypothesized that the immature ferret would display a similar sequence of brain development [both gray (GM) and white matter (WM)] to that of the preterm human infant. We describe postnatal ferret neurodevelopment with conventional and diffusion MRI. The ferret is born lissencephalic with a thin cortical plate and relatively large ventricles. Cortical folding and WM maturation take place during the first month of life. From the mid-second through the third week of postnatal life, the ferret brain undergoes a similar, though less complex, pattern of maturational changes to those observed in the human brain during the second half of gestation. GM anisotropy decreases rapidly in the first $3 \mathrm{wks}$ of life, followed by an upward surge of surface folding and WM anisotropy over the next 2 wks. (Pediatr Res 66: 80-84, 2009)
\end{abstract}

$\mathrm{P}$ reterm birth is a major public health challenge in the developed world with a high rate of neurodevelopmental impairments displayed in surviving children (1). The precise "lesion(s)" responsible for these impairments are not yet fully understood and will likely be characterized through a combination of human and animal studies.

Numerous immature animal models, including the rat, mouse, rabbit, lamb, and baboon, have been studied in relationship to the mechanisms and patterns of brain injury and altered brain development (2-8). The ferret is unique among these models in that the ferret is born lissencephalic, with cortical folding and white matter (WM) maturation taking place during the first month of life. The advantages of the ferret model include: i) a long period of forebrain neurodevelopment, facilitating high temporal resolution of brain development during vulnerable intervals; ii) complex gyral folding, early myelination, and cortical maturation that occur postnatally; iii) body size that is compatible with small animal MRI scanners, but still large enough to easily administer interventions; and iv) lower cost than larger immature animal models that require intensive care support after preterm delivery. The

Received August 13, 2008; accepted February 18, 2009.

Correspondence: Terrie Inder, M.D., Department of Neurology, Washington University, 660 South Euclid Avenue, Campus Box 8116, St. Louis, MO 63110; e-mail: inder_t@kids.wustl.edu

Supported by Green Foundation (J.J.N.), National Science Foundation Grant DMS0540701 (P.V.B.), AAP Marshall Klaus Perinatal Research Grant (A.R.B.). ferret has been used to study brain development for more than two decades, with most early studies focusing on development of the visual system (9). More recent works have characterized behavioral changes during ferret development $(10,11)$.

To enhance our understanding of the normal sequence of brain development in the immature ferret, we aimed to characterize postnatal neurodevelopment with conventional and diffusion MRI. The knowledge gained from MRI in neurodevelopmentally complex animal models, like the ferret, will facilitate the translation of basic science research to clinical application in preterm infants at risk for neurodevelopmental impairment. We hypothesized that the immature ferret would display a similar sequence of brain development [both gray matter (GM) and $\mathrm{WM}$ ] to that of the preterm human infant. Previous work using conventional MRI of the neonatal ferret has illuminated key neuroanatomical changes during gyrogenesis (12). The addition of diffusion tensor imaging and analyses using cortical surface models complement conventional MRI and allow further insights into structural and microstructural changes that occur during early brain development.

\section{METHODS}

Subjects. Ferret litters were bred by and obtained from a commercial vendor (Marshall BioResources, North Rose, NY) after a 41-d gestation. They were delivered to a dedicated animal facility at Washington University on postnatal d 3 (P3) or later. Brains were obtained via extraction after sacrifice at carefully timed ages. Each ferret brain was fixed by intracardiac perfusion with $4 \%$ paraformaldehyde ( $\mathrm{pH} 7.4)$ after clearing the vascular system with $0.1 \mathrm{M}$ PBS containing heparin ( 3 units $/ \mathrm{mL}$ ). After extraction, the brains were placed in $4 \%$ paraformaldehyde and stored at $4{ }^{\circ} \mathrm{C}$. Thirty-five ferret brains were fixed and imaged. The ferret brains were aimed to be spread across early development (up to P35) with more kits at the weekly intervals in the first 3 wk of life at P7-8, P14-15, and P20-21. Additionally, 32 in vivo image acquisitions were performed to evaluate longitudinal changes in individual ferrets before sacrifice. Procedures were reviewed by the Washington University Animal Studies Committee and performed in accordance with the Animal Welfare Act and the National Institutes of Health Guide for the Care and Use of Laboratory Animals.

In Vitro MR image acquisition. Spin-echo images were acquired of 35 fixed ferret brains from P4 through adulthood, with P4 $(n=2), \mathrm{P} 6(n=2), \mathrm{P} 8$ $(n=5), \mathrm{P} 10(n=2), \mathrm{P} 13(n=1), \mathrm{P} 15(n=3), \mathrm{P} 17(n=1), \mathrm{P} 20(n=2), \mathrm{P} 21$ $(n=4), \mathrm{P} 24(n=1), \mathrm{P} 28(n=2), \mathrm{P} 31(n=1), \mathrm{P} 37(n=1), \mathrm{P} 59(n=1)$, P66 $(n=1)$, P83 $(n=1)$, adult $(n=5)$. Imaging was performed using a $33-\mathrm{cm}$ clear bore, 4.7-T magnet controlled by a Varian INOVA console. Data were acquired using single-turn RF coils matched in size to each brain. The

\footnotetext{
Abbreviations: ADC, apparent diffusion coefficients; E, embryonic day; GM, gray matter; P, postnatal day; RA, relative anisotropy; SFI, surface folding index; WM, white matter
} 
brains were placed in modified $6,10,20,35$, and $60 \mathrm{~mL}$ syringes, corresponding to brain size, and immersed in $4 \%$ phosphate-buffered paraformaldehyde during imaging.

Contrast among GM, WM, and cerebrospinal fluid (CSF) in conventional MR images is generally provided by differences in the time constants of transverse relaxation, (T2, spin-spin relaxation time), and longitudinal relaxation ( $\mathrm{T} 1$, spin-lattice relaxation time). In the developing brain, these time constants change as the brain matures due to changes in water content and myelination (13). In this study, brain tissue $\mathrm{T} 2$ values were measured at $4.7 \mathrm{~T}$ in both GM and WM by measuring signal intensity as a function of echo time (TE) using a Hahn spin echo approach. The data points were fit to an exponential curve (14). The T2 time constant decreases with age.

$\mathrm{T} 2$-weighted images were acquired with $\mathrm{TE} \approx$ the average $\mathrm{T} 2$ from both GM and WM determined for that age to optimize contrast-to-noise ratio in the images. Voxel size was gradually increased as total brain size increased, from $150 \times 150 \times 150 \mu \mathrm{m}$ (isotropic) at P4 to $350 \mu \mathrm{m}$ isotropic in the adult.

Diffusion weighted images were acquired with acquisition parameters chosen for immature brains $(<5 \mathrm{wk}$ old $)$ : $\mathrm{TE}=67 \mathrm{~ms}$, TR $=3.4-5.8 \mathrm{~s}$, 250-300 $\mu \mathrm{m}$ isotropic voxel size, diffusion gradient duration $=9.5 \mathrm{~ms}$, diffusion gradient amplitude $=20 \mathrm{Gauss} / \mathrm{cm}$, and delay between diffusion gradients $=50 \mathrm{~ms}\left(b=200-12,100 \mathrm{~s} / \mathrm{mm}^{2}\right)$. The diffusion gradient orientation was varied over 25 directions (15). A reference image was obtained with $b=0 \mathrm{~s} / \mathrm{mm}^{2}$. For more mature brains $(>5 \mathrm{wk}$ old), the spin-spin relaxation time constant was shorter, and therefore acquisition parameters were $\mathrm{TE}=35 \mathrm{~ms}, 250-350 \mu \mathrm{m}$ isotropic voxel size, $b=2700 \mathrm{~s} / \mathrm{mm}^{2}$, and gradient orientation was varied over 22 directions.

In Vivo MR image acquisition. We obtained MR images on 32 separate occasions on live ferrets (P4-adult). Seven kits were serially imaged, with one kit scanned weekly from P7 through 42. Each ferret was initially anesthetized with $2-4 \%$ isoflurane in a vented anesthesia chamber. After induction of anesthesia, the snout was placed in a nose cone with an attached palate bar or tooth bar (if teeth were present). Anesthesia was subsequently maintained via the nose cone with $1-2 \%$ isoflurane in $\mathrm{O}_{2}(1.5 \mathrm{~L} / \mathrm{min})$. The animal was held still in the prone position secured by molded head supports. The animal's pulse rate and oxygen saturation were monitored with an MRI-compatible pulse-oximeter (Nonin Medical, Plymouth, MN) attached to a hind paw. Body temperature was maintained with circulating warm water. The entire assembly (animal, restraint, water, oximeter probe, and RF coils) was contained in a plastic bed inserted in the bore of the MR scanner (Fig. 1).
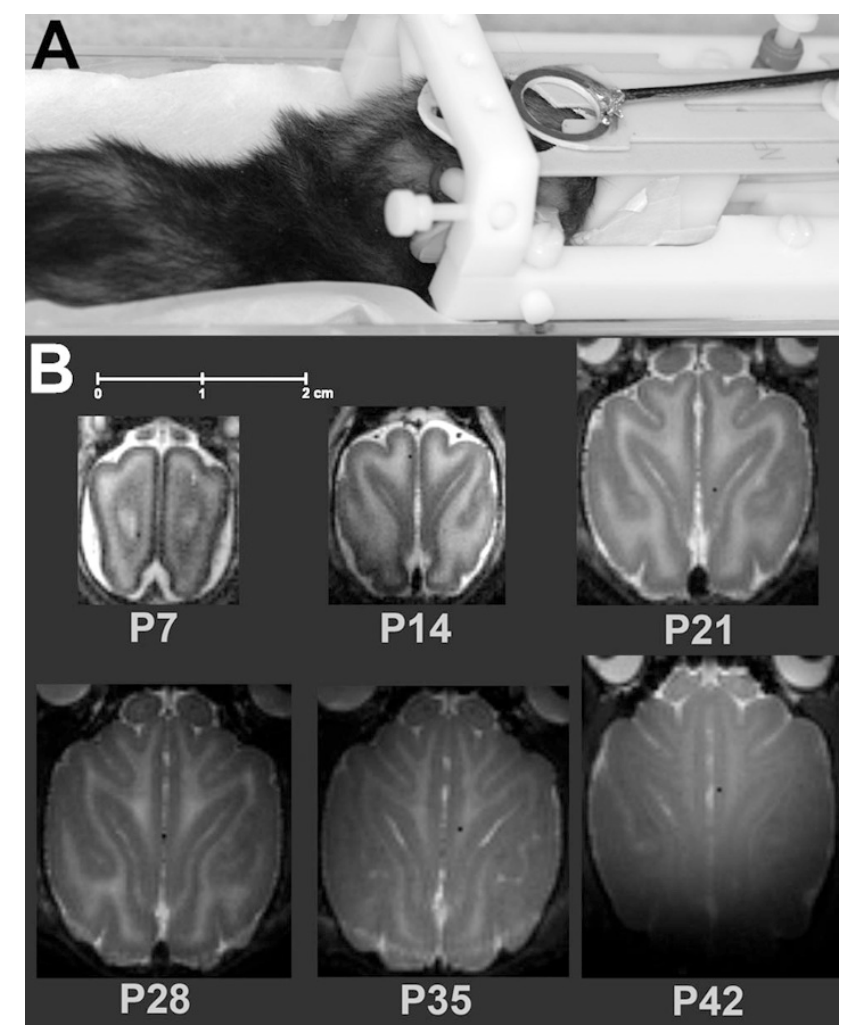

Figure 1. (A) In vivo imaging apparatus with postnatal d 28 (P28) male ferret. (B) T2-weighted horizontal MR slices at the level of the centrum semiovale in a male ferret imaged weekly from P7 through P42.
Anesthesia time ranged from 150 to $250 \mathrm{~min}$. Images were acquired using an 11.7-T magnet controlled by a Varian INOVA console with a two-coil system consisting of a surface receive coil placed just above the skull and a volume-type transmit coil. Receive coil sizes were matched to the ferret brains to optimize signal-to-noise ratio. Serially imaged kits were returned to their mothers following recovery from anesthesia.

MR image processing. Conventional T2-weighted images were qualitatively evaluated for developmental landmarks in the white and GM. Surface folding index (SFI) was calculated after the method of Dieni et al. (4). Using T2-weighted images, the pial boundaries of the cerebral cortex of horizontal slices at the level of the mid-caudate (excluding basal ganglia and diencephalon) were traced using Adobe Photoshop CS3 Extended version 10.0.1 (Adobe Systems Incorporated, San Jose, CA). The length of the pial boundary $(L)$ was measured, as was the area $(A)$ enclosed by that boundary. The SFI was calculated using equation 1 (note that a normalization constant, $(4 \pi)^{-1}$, was used so that a circle has an SFI of 1).

$$
\mathrm{SFI}=\frac{L^{2}}{4 \pi \times A}
$$

Raw data from diffusion studies were phase-corrected using Bayesian probability analysis (16) so that noise was Gaussian and centered about zero. A local diffusion tensor was estimated for each voxel, and relative anisotropy (RA) and apparent diffusion coefficient (ADC) were derived from the SD (RA) and mean (ADC) of the eigenvalues of this diffusion tensor. Regions of interest (ROIs) were placed on horizontal slices in the WM and GM lateral to the caudate nucleus, and in the corpus callosum and anterior commissure using Analyze version 7.0 (Rochester, MN). ROIs were also placed in the caudate nucleus and in frontal, somatosensory, and occipital WM tracts on horizontal slices superior to the level of the caudate nucleus.

Analysis of cortical surfaces provides a way to describe spatial variations in geometry and microstructure. Using Caret software (http://brainvis.wustl.edu/caret) (17), we generated surface-based renderings (triangular faces and their vertices defined in 3D space) from T2-weighted image volumes. RA estimated on each voxel was mapped onto the generated surface. Curvature was estimated by fitting a second-order polynomial to the surface near each vertex. The true and exposed cortical surface areas were determined using Caret (18). Mid-cortical and CSF/cortical GM tracings were used to determine true surface area for fixed and in vivo brains, respectively. Area ratios were calculated from these surface areas to quantify the extent of cortical packing for 10 fixed brains (P4, 6, 10, 13, 17, 21, 31, 66, and 2 adults) and four sets of in vivo images of a single ferret kit imaged on P7, 14, 21, and 28.

\section{RESULTS}

Gross examination. The P4 ferret brain is nearly lissencephalic and bright white. The cortex undergoes complex folding during the first $4 \mathrm{wk}$ of postnatal life. In the fifth and sixth weeks of life, as kits are weaned, cortical tissue continues to increase in volume, but also darkens slightly, progressing toward the color of light brown found in the adult brain. Additionally, from the fifth week to adulthood the brain's shape changes. The rostral portion extends forward and narrows, the gyri become less rounded, and the sulcal spaces narrow. Brain weight increases linearly over the first $5 \mathrm{wk}$ of life and then begins to plateau (Fig. 2). There is no notable difference in size or shape of the body or brain between males and females in the first 6 wk of life during the period of our major observations.

Conventional imaging. The youngest ferret imaged (P4) displayed a simple, smooth, thin cortical plate. The ventricles were relatively large and the subventricular zone appeared prominent (Fig. 3). At P10, the cortex displayed simple folding with the signal in both the white and GM becoming more homogeneous. The volume of the ventricles decreased slightly, and the subventricular zone remained prominent. By P17, the cortex appeared thicker and more complex with widespread folding and the ventricles and the subventricular zone were difficult to visualize. 

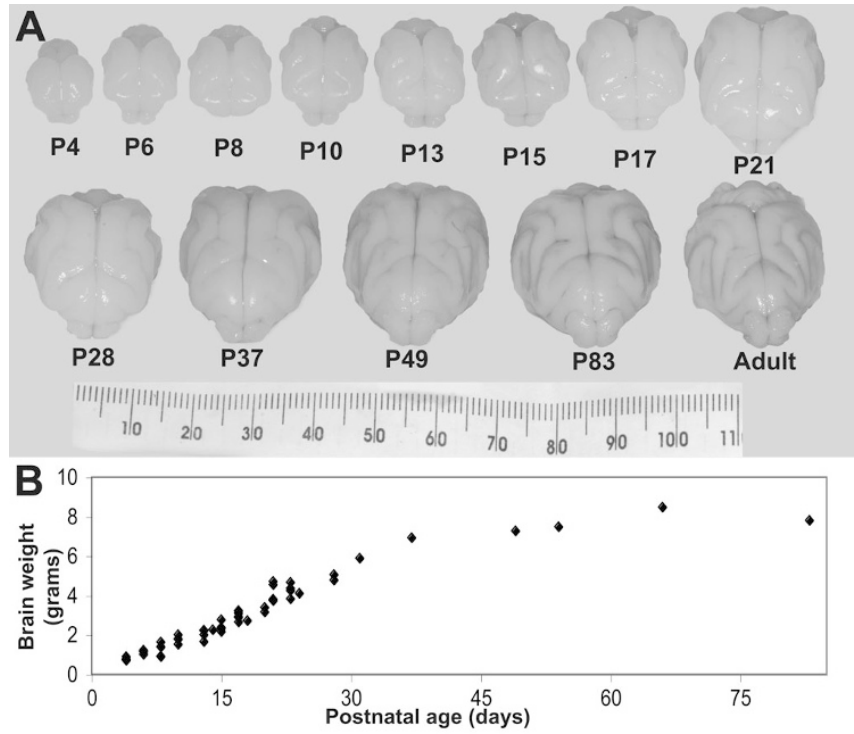

Figure 2. (A) Photographs of fixed ferret brains from postnatal d 4 (P4) through adulthood. $(B)$ Plot of brain weights from P4-83.

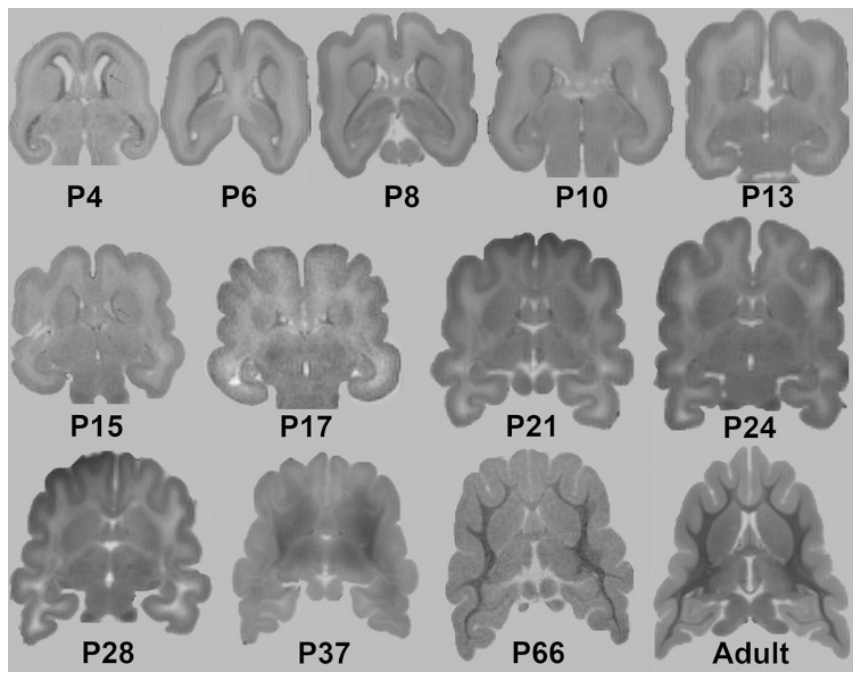

Figure 3. T2-weighted horizontal mid-caudate level MR slices of fixed ferrets from postnatal d 4 (P4) through adulthood. Note the increase in cortical folding occurring before myelination. The reversal in image intensity occurs at P37.

At P21, T2 hyperintensity appeared within the maturing region of the caudal WM and T2 hypointensity, consistent with areas of myelination, emerged within the posterior limb of the internal capsule and centrum semiovale. Over the after week, T2 hyperintensity extended rostrally while regions of T2 hypointensity (myelination) expanded throughout the cerebral WM. At P37, gray/white contrast diminished, emerging into a reversal of T2 contrast characteristics. Thus, cortical gray matter became hyperintense, the deep nuclear gray matter remained hypointense, and the sulcal depths increased and became narrower. Over the next week (P42) gray/white contrast fully reversed compared with the immature brain. From then, myelination within the WM continued to become more hypo-intense into adulthood with caudal brain narrowing.
Quantitatively, the SFI increased gradually over the first 3 wk of postnatal life, then rapidly during the fourth and fifth weeks of life.

Diffusion weighted imaging. During the first $21 \mathrm{~d}$ of postnatal life, ferret cortical gray matter anisotropy rapidly decreased, with minor increases in WM anisotropy. An upward surge in WM anisotropy occurred from 21 to $35 \mathrm{~d}$ of age. Subsequent changes in WM anisotropy occurred more slowly (Fig. 4).

Surface-based analysis. The mean curvature values and area ratios calculated with CARET software (17) are depicted in Figures $5 A$ and $B$, respectively. Minimal curvature is present at $\mathrm{P} 4$. The cortical curvature increases during each of the first 4 wks of development, as shown at P10, 17, and 24 in Figure $5 \mathrm{~A}$. The area ratio, a measure of cortical packing, increases linearly for the first 4 wks of life, then begins to plateau.

Comparison with human development. From these preliminary data, it appears that the ferret brain undergoes a similar (though less complex) sequence of development between postnatal d 10 and 21 to the preterm human brain between 25

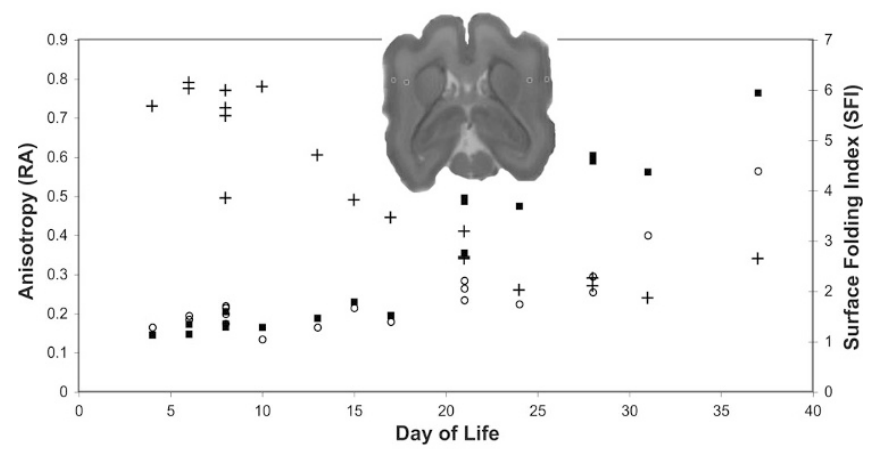

Figure 4. Diffusion anisotropy in cortical gray $(+)$ and white $(\bigcirc)$ matter, along with the surface-folding index ( $\square$ ) in ferrets from postnatal d 4 through 37. Note the earliest change is a decrease in gray matter occurring before the increase in WM anisotropy. Surface folding parallels the change in WM anisotropy. The locations of regions of interest used for anisotropy are shown in a horizontal slice from a P8 brain

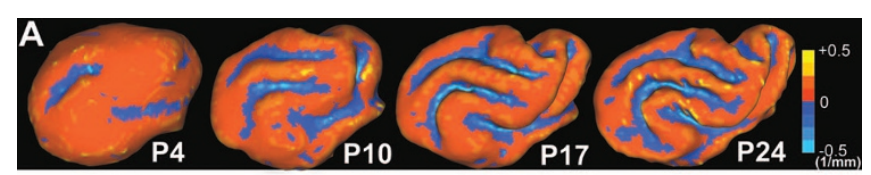

B

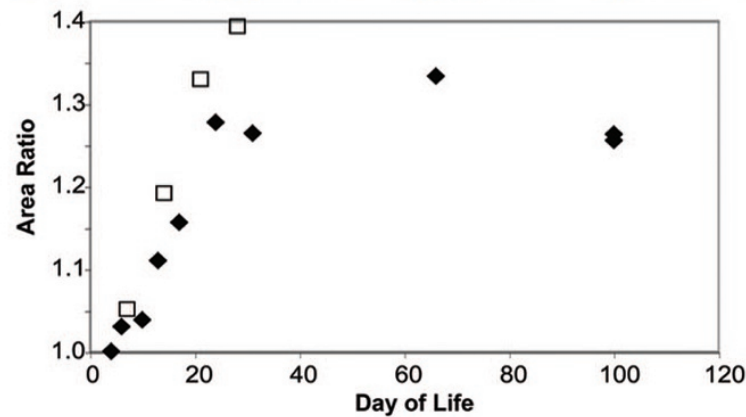

Figure 5. (A) Curvatures are mapped on $3 \mathrm{D}$ cortical surfaces at postnatal $\mathrm{d}$ $4,10,17$, and 24. (B) Area ratios quantify the degree of cortical packing in 10 fixed $(\checkmark)$ and 4 in vivo $(\square)$ ferret brains from postnatal $\mathrm{d} 4$ through adulthood and postnatal d 7 through 28 , respectively. 


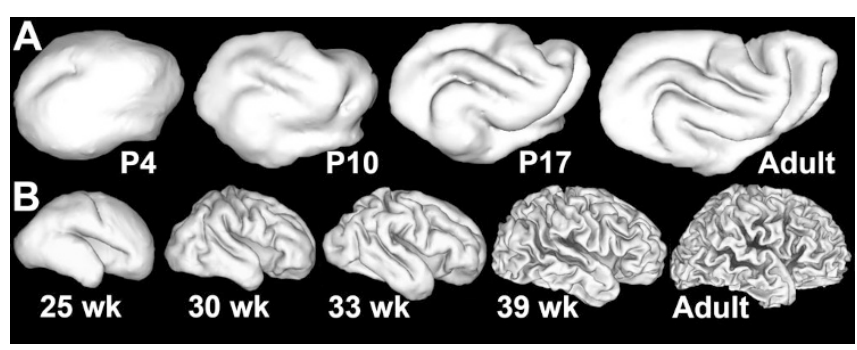

Figure 6. 3D reconstructions of $(A)$ postnatal d 4, 10, 17, and adult ferret brains and $(B)$ human brains at $25,30,33$, and 39 wk gestation and adult maturation.

and 40 wk gestation. Representative 3D reconstructions of ferret cortical surfaces, together with human cortical surface representations at comparable stages are shown in Figure 6.

\section{DISCUSSION}

The MR data from our study demonstrate that despite the human brain being far more complex than the ferret brain, the pattern of changes has both qualitative and quantitative similarities. These data confirm the value of the immature ferret as a model for studies of brain injury and development, relevant to both the preterm and term-born human infant. In addition, MRI can be an important translational tool between the human and animal model.

There are notable variations in the number of ferret kits that are imaged at each time point. Because of kit availability, higher numbers of subjects were imaged at some time points with a single kit at others. However, as the ferret kit brains displayed smooth and consistent transitions during early development, extrapolations of observations between the ages was felt to be appropriate.

Comparison of MRI features with histology during ferret brain development. Production of the neurons and glia of the ferret forebrain cortex takes place in the subventricular zone, with neurons and glia migrating to the developing cortical plate. Maturation of individual cortical layers generally proceeds with an inside to outside (layer VI to layer II) sequence. Maturation also proceeds in a rostrocaudal and laterodorsal direction $(19,20)$. Cortical neuron production begins on approximately embryonic d 20 (E20) and continues for $5 \mathrm{wk}$ (21). By E29 neurons are present along the entire cortical plate surface and somatosensory neurogenesis completes rostrally by E36 (19). Histologically, neurogenesis in the somatosensory cortex is complete by $\mathrm{P} 2$, whereas neuron production in the visual cortex continues through the second week of postnatal life (22).

The major features of postnatal brain maturation observed on MRI in the ferret begin with early development (P4-10), characterized by commencement of gyral folding, loss of cortical GM anisotropy, and moderate increases in curvature. The next phase in development (P10-28) is characterized on MRI by marked increases in WM anisotropy, SFI, and curvature. These maturational changes in the second and third postnatal weeks of ferret life are similar to the changes occurring in second half of human gestation (23). The layers of cortex become increasingly histologically distinct over the first 4 wks of life (22). The decrease in signal intensity of the cortical ribbon on T2-weighted images in the fourth week of life corresponds temporally with the neuronal cell differentiation into larger, less densely spaced cells observed in previous histologic examinations $(12,19,20)$.

MRI also delineates a third phase of neurodevelopment in this model, which occurs from the fifth to seventh week of postnatal life. During this phase, myelination and a reversal of gray/white contrast on T2-weighted images occur, accompanied by no major changes in the SFI, curvature, anisotropy, or brain size. The decrease in WM signal intensity on T2weighted imaging, occurring in the fifth and sixth weeks of life, corresponds to maturation of myelin. By 2 mo of age, cortical cytoarchitecture closely resembles its adult form (21). After this phase, the brain elongates rostrally, the sulcal spaces continue to narrow, and the gyri become less rounded and more squarely shaped, but no significant changes of our measured indices occur.

This long interval of neuromaturation is in contrast to the rapid maturation of rodents (21).

Application of MRI in human and immature animal models. MRI studies in the prematurely born infant have documented common, qualitative abnormalities, including WM signal abnormalities, ventriculomegaly, and thinning of the corpus callosum (24). These abnormalities, when present at term equivalent, have been correlated with adverse neurodevelopmental outcomes at 2 y of age (25). Additionally, diffusion weighted imaging has been shown to reflect WM and GM microstructural maturation in human brain from $26 \mathrm{wk}$ gestation through infancy. The two quantitative measures from diffusion imaging that have been shown to be sensitive to brain injury in both the acute and chronic phases in preterm (26) and term-born infants $(27,28)$ are ADC and RA. Another quantitative measure, SFI, quantifies the extent of gyral folding and has been used as a marker for injury and development in animal models (29). Surface-based morphometry was recently used to quantify changes in gyrification occurring in preterm human infants from 26 to $36 \mathrm{wk}$ gestation (30). Thus, a combination of these analysis techniques was undertaken in this study.

Conventional MRI methods have been applied to several immature animal models, including baboons, lambs, rabbits, rats, ferrets, and mice expanding our knowledge of conventional MRI characteristics of brain development and injury $(2-4,8,12)$. However, apart from the rabbit (31), baboon (7), and rodent (32), few quantitative measures have been made in these studies. Normative values of anisotropy and diffusivity are currently used to assess for the presence and degree of brain injury. Application of these quantitative measures to both human and animal studies can enhance the translational understanding of the model with the human disease process.

Future of the ferret model of neurodevelopment. The future of the ferret as a neurodevelopmental animal model remains promising. This model displays complex gyral folding and myelination, a long interval of neurodevelopment, and numerous similarities to the preterm human brain. We are currently developing an injury model using chronic hypoxia to generate mild, diffuse patterns of brain injury. Additionally, 
behavioral testing with water mazes will strengthen our understanding of both development and injury. Future analysis will combine functional neurodevelopmental testing (water maze performance) with both imaging and histologic markers of development and injury. The immature ferret model can be used to expand our knowledge about the effects of current and future interventions used in the care of neonates on brain development and injury. Improvements in understanding these effects and the mechanisms leading to brain injury in preterm infants will help us refine neonatal care to limit the frequency and severity of adverse neurologic outcomes.

\section{REFERENCES}

1. Moster D, Lie RT, Markestad T 2008 Long-term medical and social consequences of preterm birth. N Engl J Med 359:262-273

2. Back SA, Riddle A, Hohimer AR 2006 Role of instrumented fetal sheep preparations in defining the pathogenesis of human periventricular white-matter injury. J Child Neurol 21:582-589

3. Derrick M, Drobyshevsky A, Ji X, Tan S 2007 A model of cerebral palsy from fetal hypoxia-ischemia. Stroke 38:731-735

4. Dieni S, Inder T, Yoder B, Briscoe T, Camm E, Egan G, Denton D, Rees S 2004 The pattern of cerebral injury in a primate model of preterm birth and neonatal intensive care. J Neuropathol Exp Neurol 63:1297-1309

5. Duncan JR, Cock ML, Scheerlinck JP, Westcott KT, McLean C, Harding R, Rees SM 2002 White matter injury after repeated endotoxin exposure in the preterm ovine fetus. Pediatr Res 52:941-949

6. Hagberg H, Bona E, Gilland E, Puka-Sundvall M 1997 Hypoxia-ischaemia model in the 7-day-old rat: possibilities and shortcomings. Acta Paediatr Suppl 422:85-88

7. Kroenke CD, Van Essen DC, Inder TE, Rees S, Bretthorst GL, Neil JJ 2007 Microstructural changes of the baboon cerebral cortex during gestational development reflected in magnetic resonance imaging diffusion anisotropy. J Neurosci 27:12506-12515

8. Lodygensky GA, Inder TE, Neil JJ 2008 Application of magnetic resonance imaging in animal models of perinatal hypoxic-ischemic cerebral injury. Int J Dev Neurosci 26:13-25

9. Katz LC, Crowley JC 2002 Development of cortical circuits: lessons from ocular dominance columns. Nat Rev Neurosci 3:34-42

10. Christensson M, Garwicz M 2005 Ontogenesis of within-session locomotor habituation in the open field. Neuroreport 16:1319-1323

11. Christensson M, Garwicz M 2005 Time course of postnatal motor development in ferrets: ontogenetic and comparative perspectives. Behav Brain Res 158:231-242

12. Neal J, Takahashi M, Silva M, Tiao G, Walsh CA, Sheen VL 2007 Insights into the gyrification of developing ferret brain by magnetic resonance imaging. J Anat 210:66-77
13. Martin E, Kikinis R, Zuerrer M, Boesch C, Briner J, Kewitz G, Kaelin P 1988 Developmental stages of human brain: an MR study. J Comput Assist Tomogr 12:917-922

14. Hahn EL 1950 Spin Echoes. Phys Rev 80:580-594

15. Batchelor PG, Atkinson D, Hill DL, Calamante F, Connelly A 2003 Anisotropic noise propagation in diffusion tensor MRI sampling schemes. Magn Reson Med 49:1143-1151

16. Neil JJ, Bretthorst GL 1993 On the use of Bayesian probability theory for analysis of exponential decay data: an example taken from intravoxel incoherent motion experiments. Magn Reson Med 29:642-647

17. Van Essen DC, Drury HA, Dickson J, Harwell J, Hanlon D, Anderson CH 2001 An integrated software suite for surface-based analyses of cerebral cortex. J Am Med Inform Assoc 8:443-459

18. Van Essen DC 2005 A Population-Average, Landmark- and Surface-based (PALS) atlas of human cerebral cortex. Neuroimage 28:635-662

19. McSherry GM, Smart IH 1986 Cell production gradients in the developing ferret isocortex. J Anat 144:1-14

20. Smart IH, McSherry GM 1986 Gyrus formation in the cerebral cortex of the ferret II. Description of the internal histological changes. J Anat 147:27-43

21. Jackson CA, Peduzzi JD, Hickey TL 1989 Visual cortex development in the ferret I. Genesis and migration of visual cortical neurons. J Neurosci 9:1242-1253

22. Noctor SC, Scholnicoff NJ, Juliano SL 1997 Histogenesis of ferret somatosensory cortex. J Comp Neurol 387:179-193

23. McKinstry RC, Mathur A, Miller JH, Ozcan A, Snyder AZ, Schefft GL, Almli CR, Shiran SI, Conturo TE, Neil JJ 2002 Radial organization of developing preterm human cerebral cortex revealed by non-invasive water diffusion anisotropy MRI. Cereb Cortex 12:1237-1243

24. Inder TE, Warfield SK, Wang H, Huppi PS, Volpe JJ 2005 Abnormal cerebral structure is present at term in premature infants. Pediatrics 115:286-294

25. Woodward LJ, Anderson PJ, Austin NC, Howard K, Inder TE 2006 Neonatal MRI to predict neurodevelopmental outcomes in preterm infants. N Engl J Med 355:685694

26. Counsell SJ, Allsop JM, Harrison MC, Larkman DJ, Kennea NL, Kapellou O, Cowan FM, Hajnal JV, Edwards AD, Rutherford MA 2003 Diffusion-weighted imaging of the brain in preterm infants with focal and diffuse white matter abnormality. Pediatrics 112:1-7

27. McKinstry RC, Miller JH, Snyder AZ, Mathur A, Schefft GL, Almli CR, Shimony JS, Shiran SI, Neil JJ 2002 A prospective, longitudinal diffusion tensor imaging study of brain injury in newborns. Neurology 59:824-833

28. Ward P, Counsell S, Allsop J, Cowan F, Shen Y, Edwards D, Rutherford M 2006 Reduced fractional anisotropy on diffusion tensor magnetic resonance imaging after hypoxic-ischemic encephalopathy. Pediatrics 117:e619-e630

29. Rees S, Stringer M, Just Y, Hooper SB, Harding R 1997 The vulnerability of the fetal sheep brain to hypoxemia at mid-gestation. Brain Res Dev Brain Res 103:103118

30. Dubois J, Benders M, Cachia A, Lazeyras F, Ha-Vinh Leuchter R, Sizonenko SV Borradori-Tolsa C, Mangin JF, Huppi PS 2008 Mapping the early cortical folding process in the preterm newborn brain. Cereb Cortex 18:1444-1454

31. Drobyshevsky A, Song SK, Gamkrelidze G, Wyrwicz AM, Derrick M, Meng F, Li L, Ji X, Trommer B, Beardsley DJ, Luo NL, Back SA, Tan S 2005 Developmental changes in diffusion anisotropy coincide with immature oligodendrocyte progression and maturation of compound action potential. J Neurosci 25:5988-5997

32. Sun SW, Neil JJ, Song SK 2003 Relative indices of water diffusion anisotropy are equivalent in live and formalin-fixed mouse brains. Magn Reson Med 50:743-748 